\title{
STACEE Observations of Markarian 421 During an Extended Gamma-Ray Outburst
}

\author{
L. M. Boone ${ }^{1}$, J. A. Hinton ${ }^{2,11}$, D. Bramel ${ }^{3}$, E. Chae ${ }^{2}$, C. E. Covault ${ }^{4}$, P. Fortin ${ }^{5}$, \\ D. M. Gingrich ${ }^{6,7}$, D. S. Hanna ${ }^{5}$, R. Mukherjee ${ }^{3}$, C. Mueller ${ }^{5}$, R. A. Ong ${ }^{8}$, K. Ragan ${ }^{5}$, \\ R. A. Scalzo ${ }^{2}$, D. R. Schuette ${ }^{8,9}$, C. G. Théoret ${ }^{5,10}$, and D. A. Williams ${ }^{1}$
}

\begin{abstract}
The active galaxy Markarian 421 underwent a substantial outburst in early 2001. Between January and May of that year, the STACEE detector was used to observe the source in $\gamma$-rays between the energies of 50 and $500 \mathrm{GeV}$. These observations represent the lowest energy $\gamma$-ray detection of this outburst by a ground-based experiment. Here we present results from these observations, which indicate an average integral $\gamma$-ray flux of $(8.0 \pm 0.7 \pm 1.5) \times 10^{-10} \mathrm{~cm}^{-2} \mathrm{~s}^{-1}$ above $140 \mathrm{GeV}$. We also present a light curve for Markarian 421 as observed by STACEE from March to May, and compare our temporal, as well as spectral, measurements to those of other experiments.
\end{abstract}

Subject headings: galaxies: active-BL Lacertae objects: individual (Markarian 421) — gamma rays: observations

\footnotetext{
${ }^{1}$ Santa Cruz Institute for Particle Physics, University of California, Santa Cruz, CA 95064, USA

${ }^{2}$ Enrico Fermi Institute, University of Chicago, 5640 Ellis Ave., Chicago, IL 60637, USA

${ }^{3}$ Columbia University \& Barnard College, New York, NY 10027, USA

${ }^{4}$ Department of Physics, Case Western Reserve University, 10900 Euclid Ave., Cleveland, OH 44106, USA

${ }^{5}$ Department of Physics, McGill University, Montreal, Quebec H3A 2T8, Canada

${ }^{6}$ Centre for Subatomic Research, University of Alberta, Edmonton, Alberta T6G 2N5, Canada

${ }^{7}$ TRIUMF, Vancouver, British Columbia V6T 2A3, Canada

${ }^{8}$ Department of Physics \& Astronomy, University of California, Los Angeles, CA 90095, USA

${ }^{9}$ Present address: Department of Physics, Cornell University, Ithaca, NY 14853

${ }^{10}$ Present address: Laboratoire de Physique Corpusculaire et Cosmologie, Collège de France, F-75231 Paris CEDEX 05, France

${ }^{11}$ Present address: Max-Planck-Institut für Kernphysik Postfach 1039 80, D-69029 Heidelberg, Germany
} 


\section{Introduction}

The blazar Markarian 421 is one of only a handful of astrophysical objects detected in the very high energy (VHE) $\gamma$-ray regime, between $10 \mathrm{GeV}$ and $100 \mathrm{TeV}$. At a red-shift of 0.031, it is the closest BL Lac object seen by EGRET, and the first to be detected by a ground-based TeV instrument (Punch et al. 1992; Petry et al. 1996). Along with Markarian $501(\mathrm{z}=0.034)$, it is one of the most prominent sources of extra-galactic VHE $\gamma$-rays, and has been regularly monitored by atmospheric Cherenkov telescopes since its TeV identification (see Catanese \& Weekes 1999, for a review).

Markarian 421 is an X-ray selected BL Lac object, and as such, it exhibits the doublehumped spectrum that is characteristic of blazars (e.g. Takahashi et al. 2000). The lower energy hump, believed to arise from synchrotron radiation, peaks in the X-ray range, while the higher energy hump, generally attributed to inverse Compton (IC) scattering of soft photons, peaks in the GeV to TeV energy range.

The details of the emission processes that produce the IC hump are, as yet, unresolved. For example, the origin of the soft photons that seed the IC component of the spectrum is an outstanding question in the study of blazars. Although synchrotron self-Compton models, in which the seed photons originate from synchrotron radiation within the blazar jets, are generally favored for Markarian 421 (Coppi 1992; Coppi \& Aharonian 1999), other competing models exist. These include external Compton models (Dermer, Schlickeiser, \& Mastichiadis 1992; Sikora, Begelman, \& Rees 1994), proton-induced cascades (Mannheim 1993), and proton synchrotron models (Aharonian 2000; Mücke \& Protheroe 2001).

Markarian 421 has been given to strong periods of flaring activity over the past few years. Of note was a flare in 1996 during which the source was detected at more than ten times the flux of the Crab Nebula (Gaidos et al. 1996). In the early part of 2001, a rather impressive flare was again observed (Boerst et al. 2001), with reported TeV fluxes of comparable magnitude to those recorded in 1996, but of much longer duration (Krennrich et al. 2001).

It was during the 2001 flare that STACEE-48, an intermediate incarnation of STACEE (the Solar Tower Atmospheric Cherenkov Effect Experiment), was commencing operation. STACEE was able to observe the activities of Markarian 421 for much of the flaring period. These observations are of particular note as they represent the only $\gamma$-ray detection below $200 \mathrm{GeV}$ during this flare. 


\section{The STACEE-48 Detector}

STACEE is a low threshold ground-based $\gamma$-ray detector located at the National Solar Thermal Test Facility near Albuquerque, New Mexico. STACEE uses the large steerable mirrors (heliostats) of an existing solar research facility to collect Cherenkov photons from particle cascades in the atmosphere. The photons are focused, via secondary optics located on a central tower, onto an array of photomultiplier tubes, and their arrival times are recorded and processed by high-speed electronics. Because of their very large collection areas, wavefront sampling detectors, such as STACEE and CELESTE (de Naurois et al. 2002), are currently the only ground-based instruments capable of detecting $\gamma$-rays below $200 \mathrm{GeV}$. STACEE was commissioned for its first science observations with 32 heliostats (STACEE-32, see Hanna et al. 2002) and was used to detect $\gamma$-ray emission from the Crab Nebula in 1998 and 1999 (Oser et al. 2001).

STACEE-48 (Covault et al. 2001) was an upgrade of the prior 32-channel experiment in which 16 new heliostats were added, bringing the total to 48, each with a mirror area of 37 $\mathrm{m}^{2}$. The introduction of these new heliostats had the dual advantage of increasing the total collection area, as well as producing a more favorable detector geometry. In addition, by incorporating these new channels into the trigger, the energy threshold of the experiment was lowered from $190 \mathrm{GeV}$ to $140 \mathrm{GeV}$ due to improved suppression of the night sky background.

The trigger hardware for STACEE-48 was re-instrumented with custom built modules that handle the tasks of signal delay and trigger formation (Martin \& Ragan 2000). The delays are constructed from a $125 \mathrm{MHz}$ pipeline capable of storing and delaying one hit per channel in each $8 \mathrm{~ns}$ bin. The system has a coincidence resolution of $1 \mathrm{~ns}$, by virtue of a vernier encoding scheme, and a maximum possible delay of $2048 \mathrm{~ns}$. It applies delays to each channel such that an idealized Cherenkov event will produce a coincident signal across the entire detector. Channels are grouped by geography into subclusters of eight heliostats each, and a trigger was formed if four of the six subclusters were hit within a 28 ns window. Individual subclusters registered a hit if five of their eight constituent channels fired within a 12 ns window.

Improvements from STACEE-32 were also made on a number of other fronts. We aligned the mirror facets on all 48 heliostats using a laser look-back system. The phototubes were re-calibrated, and then re-distributed in the camera to better balance the detector response. We used the full Moon to align the optics from end to end, which resulted in important improvements to the optical throughput. We also reduced the background light reflected from the asphalt on the heliostat field by up to $40 \%$ by resurfacing the field with a dark sealant. 
We have conducted extensive simulations of the STACEE-48 detector and its response to both $\gamma$-ray and hadronic events. We employ the CORSIKA air-shower simulation package (Heck et al. 1998) with the QGSJET hadronic interaction model (Kalmykov \& Ostapchenko 1993), together with custom optical and electronics simulation packages to obtain the detector response. We have been successful in reproducing various detector characteristics with the simulations, including the detector rate at zenith and the trigger rate as a function of discriminator threshold. From simulations of our effective area convolved with a $\gamma$-ray spectrum of differential index $\alpha=2.1$ (see Fig. 1), the energy threshold for our observations (defined as the peak of the detected differential spectrum) was determined to be $140 \pm 20 \mathrm{GeV}$. The error represents the shift in the peak location as the spectral index is varied between 1.7 and 2.5 .

\section{Observations}

STACEE-48 was used to observe Markarian 421 in the early part of 2001 (Boone 2002). STACEE uses an on-off technique for source observations as described in Oser et al. (2001). We take on-source data for 28 minutes, with a corresponding off-source run of 28 minutes to form an on-off pair. Our observations from March to May included about 26 nights, yielding 78 on-off pairs from 36 hours of source observations and an equal amount of background observations.

We have found that the subcluster firing rates, which are driven primarily by the nightsky background, are a powerful diagnostic of abnormal fluctuations in sky conditions. In good-quality pairs, the time evolution of the subcluster rates for both on and off-source runs are very linear, and well correlated. However, in runs with passing clouds or high haze, the rates are irregular and uncorrelated. Thus, removing runs whose subcluster rates exhibit deviations from a linear time-evolution has proven to be an effective quality selection criterion. Of the original 78 pairs, 17 were removed from the data set using this criterion.

Three on-off pairs were removed because they showed abnormally high rates from background light, and two pairs were removed due to heliostat malfunctions. Other pairs were trimmed to remove short-term contamination from an otherwise acceptable run. Removing and trimming runs reduced the usable data to 56 on-off pairs, totaling 22.0 hours of observations.

After applying all the selection criteria, the data set consists of 317, $045 \pm 563$ on-source events and 307,641 \pm 555 off-source events, yielding a 9, $404 \pm 790$ event excess in the onsource data. This corresponds to a raw average excess of $7.1 \pm 0.6$ events $/ \mathrm{min}$, where the 
quoted error is statistical only.

Of particular concern to non-imaging atmospheric Cherenkov detectors like STACEE is the relatively bright star HD 95934 (magnitude 6.16 in the B band), which is within a few

arc-minutes of Markarian 421, and well within the STACEE field of view. A calculation of the expected photon flux from this star and the trigger multiplicity required by the detector indicates that we should see much less than one false trigger per night from the star alone. However, random fluctuations in the excess noise generated on each channel by the starlight may promote an otherwise sub-threshold cosmic-ray event. Such promotions of sub-threshold background events would only occur in on-source observations, as there is no corresponding star in the off-source field of view. In this way, it may be possible to create a fake signal.

To study this star effect, we spent some time observing HIP 80460 ( $\mathrm{RA}=16.42 \mathrm{hr}$, Dec $=37.39^{\circ}$ ), a star with a magnitude and declination comparable to HD 95934. We took 12 on-off pairs, consisting of approximately five on-source hours. After applying analogous quality requirements to those described above, 11 pairs and 4.3 hours of data survive. These data indicate a raw excess of $1.9 \pm 1.2$ events/min from the star effect.

After correcting for this fake signal, the detector deadtime, and the distributions of detector configurations (as described in the next section), we detect an average $\gamma$-ray rate from Markarian 421 of $7.7 \pm 0.7 \pm 1.2 \gamma / \mathrm{min}$, where the first error is the statistical error on the Markarian 421 measurement alone, and the second represents the statistical error in the observations of HIP 80460, used to correct for the effect of the star HD 95934.

\section{Flux Determination}

Although the source is expected to be highly variable, it is useful to calculate an average flux over all the STACEE-48 observations. Detector simulations indicate that the trigger rate varies significantly as a function of zenith angle, and, to a lesser extent, detector thresholds. In order to calculate quantities that are relatively independent of these effects, we corrected the measured rate at a given detector zenith angle and threshold to the corresponding expected rate at a fiducial configuration (a zenith angle of $5^{\circ}$, near Markarian 421 transit, and a detector threshold of $\sim 5$ photoelectrons on each phototube). To do this, we simulated the detector response to an assumed source spectrum at the fiducial configuration, as well as the observation configuration. We then scaled the measured rate by the ratio of these responses to obtain a corrected constant-configuration curve.

These corrections are sensitive to assumptions about the functional form of the incident $\gamma$-ray flux. Thus, we must first choose a hypothesis for what the $\gamma$-ray spectrum might be in 
our energy range. Figure 2 is a spectral energy distribution of Markarian 421 showing measurements by EGRET, averaged over five years of observations (Hartman et al. 1999), and Whipple during the 2001 flare (Krennrich et al. 2002). The STACEE butterfly was obtained by assuming a simple power-law spectrum with a given spectral index. The normalization was then fit such that the convolution of the assumed spectrum and the detector effective area matched the actual rate measured by STACEE. This procedure was done for a range of differential spectral indices from 2.00 to 2.20, yielding a derived differential photon flux of $\left(6.4 \pm 0.5_{\text {stat }} \pm 1.3_{\text {sys }}\right) \times 10^{-9} \mathrm{~cm}^{-2} \mathrm{~s}^{-1} \mathrm{TeV}^{-1}$ at $140 \mathrm{GeV}$. Here the statistical error is the error on the Markarian 421 measurement. The systematic error is obtained by adding in quadrature the statistical error from the HIP 80460 measurement $\left(1.0 \times 10^{-9}\right)$, the variation of the result with respect to the spectral index $\left(0.5 \times 10^{-9}\right)$, and $0.6 \times 10^{-9}$ from the uncertainty in the detector corrections (see Boone 2002, for details). Note that this result represents an average for the 2001 flare. Though spectral variability was reported at higher energies (Krennrich et al. 2002; Aharonian et al. 2002), STACEE-48 was not sufficiently sensitive to resolve this variation.

Figure 3 is a plot of the correlation between the STACEE integral flux above $140 \mathrm{GeV}$ and corresponding data taken by the Whipple $\gamma$-ray telescope (Mt. Hopkins, AZ, USA) above $390 \mathrm{GeV}$ (Holder et al. 2001). Points represent data from both experiments whose time stamps agree to within 0.02 days ( $\sim 29$ minutes). A linear fit to the data yields a flux ratio of $4.3 \pm 1.4$. Assuming a simple power law spectrum for Markarian 421, this ratio corresponds to a differential spectral index of $\alpha=2.4_{-0.4}^{+0.3}$. If we include an exponential roll-off at $E_{o}=4.3 \mathrm{TeV}$, the derived index drops to $\alpha=2.3_{-0.4}^{+0.3}$, both of which are consistent with the value of $\alpha=2.14$ from Krennrich et al. (2001).

Assuming a differential spectrum of index $\alpha=2.1$ over STACEE's energy range, and after correcting for variations in the detector configuration, as well as for the observed star effect, we find the integral flux above $140 \mathrm{GeV}$ to be $\left(8.0 \pm 0.7_{\text {stat }} \pm 1.5_{\text {sys }}\right) \times 10^{-10} \mathrm{~cm}^{-2} \mathrm{~s}^{-1}$. The first error is the statistical error in the Markarian 421 observations, and the second reflects the systematic errors discussed above, of which the error in the star correction is the dominant contribution $\left( \pm 1.3 \times 10^{-10}\right)$.

\section{Temporal Analysis}

While there is a substantial excess from Markarian 421 in the entire data set, individual on-off pairs generally do not exhibit highly significant detections. By combining the observations of a single night, we can improve the flux significance at the cost of some temporal resolution. To obtain the STACEE-48 light curve for Markarian 421, we averaged the rate 
over all the observations of each night, and assigned a date which was a weighted average of the Modified Julian Date in the middle of each run.

Figure 4 includes the STACEE-48 light-curve for Markarian 421 between early March and late May. Filled circles are the counts per minute, and error bars are statistical only. For comparison, contemporaneous RXTE quick-look results (provided by the RXTE All Sky Monitor team) are plotted above, and Whipple observations (Holder et al. 2001) are plotted below. All the experiments observe an increased flux in the latter part of March, falling to lower values in April. Note that the coincident gaps in both the STACEE and Whipple coverage correspond to dates near the full Moon, during which Cherenkov telescopes generally cannot operate.

The STACEE fluxes appear correlated with both the RXTE and Whipple observations. In fact, a discrete correlation analysis (Edelson \& Krolik 1988) is consistent with the STACEE and Whipple observations being correlated on day timescales. However, the limited statistics in the nightly STACEE observations currently make analysis on shorter timescales infeasible.

\section{Conclusions}

STACEE has detected the BL Lac object, Markarian 421, with high significance in the $140 \mathrm{GeV}$ energy band, a hitherto unexplored region of its spectral energy distribution. The STACEE observed flux is consistent with other VHE $\gamma$-ray observations, and the temporal evolution of the STACEE observations appears similar to observations in both the $\mathrm{TeV}$ and $\mathrm{X}$-ray bands.

Figure 2 indicates that the STACEE measurements occupy an important region in Markarian 421's spectral energy distribution near the peak of the IC hump. Future measurements of the spectrum by STACEE, along with simultaneous data at X-ray and TeV energies should help to further constrain synchrotron and IC blazar models for this source. STACEE is currently operating with 64 channels, each equipped with a flash ADC, and should be able to obtain more detailed spectral information in the near future.

We are grateful to the staff of the National Solar Thermal Test Facility for their excellent support. Thanks to Gora Mohanty, Jeff Zweerink, Tumay Tumer, Marta Lewandowska, Scott Oser, François Vincent, and Joseph Boone. This work was supported in part by the National Science Foundation (under Grant Numbers PHY-9983836, PHY-0070927, and PHY-0296052), the Natural Sciences and Engineering Research Council, FCAR (Fonds pour la Formation de Chercheurs et l'Aide à la Recherche), the Research Corporation and the 
California Space Institute.

\section{REFERENCES}

Aharonian, F. A. 2000, New Astron., 5, 377

Aharonian, F. A., et al. 2002, A\&A, In Press

Bloom, S. D. \& Marscher, A. P. 1996, ApJ, 461, 657

Boerst, H. G., et al. 2001, IAU Circ., 7568

Boone, L. M. 2002, Ph.D. thesis, Univ. California, Santa Cruz

Catanese, M. \& Weekes, T. C. 1999, PASP, 111, 1193

Coppi, P. S. 1992, MNRAS, 258, 657

Coppi, P. S. \& Aharonian, F. A. 1999, ApJ, 521, L33

Covault, C. E., et al. 2001, in Proc 27th Int. Cosmic-Ray Conf. (Hamburg), 1, 2810

de Naurois, M., et al. 2002, ApJ, 566, 343

Dermer, C. D., Schlickeiser, R., \& Mastichiadis, A. 1992, A\&A, 256, L27

Edelson, R. A. \& Krolik, J. H. 1996, ApJ, 333, 646

Gaidos, J. A., et al. 1996, Nature, 383, L26

Hanna, D. S., et al. 2002, NIM-A, 491, 126.

Hartman, R. C., et al. 1999, ApJS, 123, 79

Heck, D., Knapp, J., Capdevielle, J. N., Schatz, G., \& Thou, T. 1998 Report FZKA 5019 Forschungszentrum Karlsruhe

Holder, J., et al. 2001, in Proc 27th Int. Cosmic-Ray Conf. (Hamburg), 1, 2613

Kalmykov, N. N. \& Ostapchenko, S. S. 1993, Yad. Fiz., 56, 105

Krennrich, F., et al. 2001, ApJ, 560, L45

Krennrich, F., et al. 2002, ApJ, 575, L9 
Mannheim, K. 1993, A\&A, 269, 67

Martin, J.-P. \& Ragan, K. 2000, in Proc. IEEE Nuclear Science Symposium (Lyon), 12, 141

Mücke, A. \& Protheroe, R. J. 2001, Astropart. Phys., 15, 121

Oser, S., et al. 2001, ApJ, 547, 949

Punch, M., et al. 1992, Nature, 358, 477

Petry, D., et al. 1996, å, 311, L13

Sikora, M., Begelman, M. C., \& Rees, M. J. 1994, ApJ, 421, 153

Takahashi, T. 2000, ApJ, 542, 105 


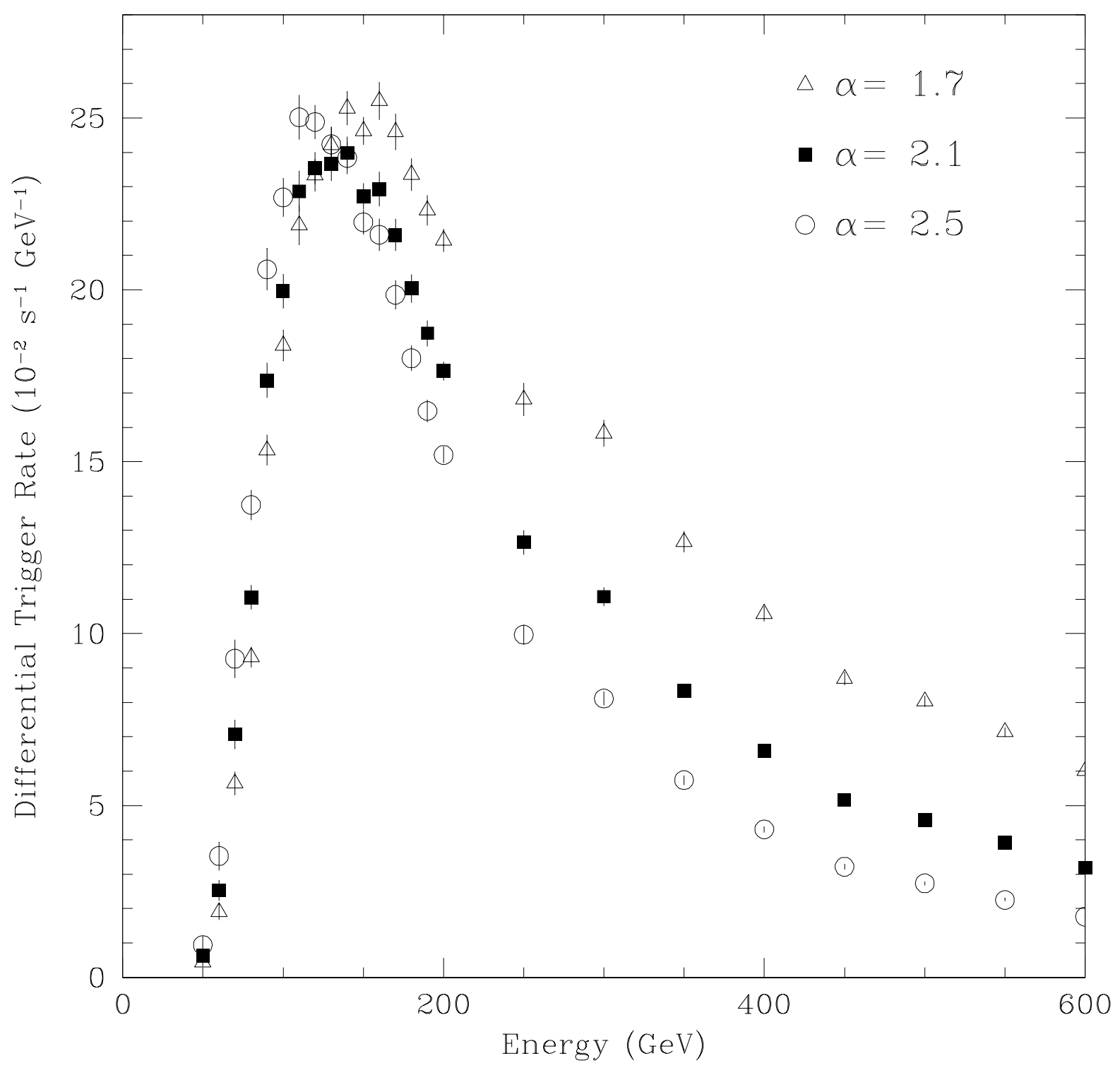

Fig. 1. - STACEE-48 detector response to several different power-law spectra. Spectra are normalized to the same incident integral flux above $50 \mathrm{GeV}$ for the given differential spectral index, $\alpha$. 


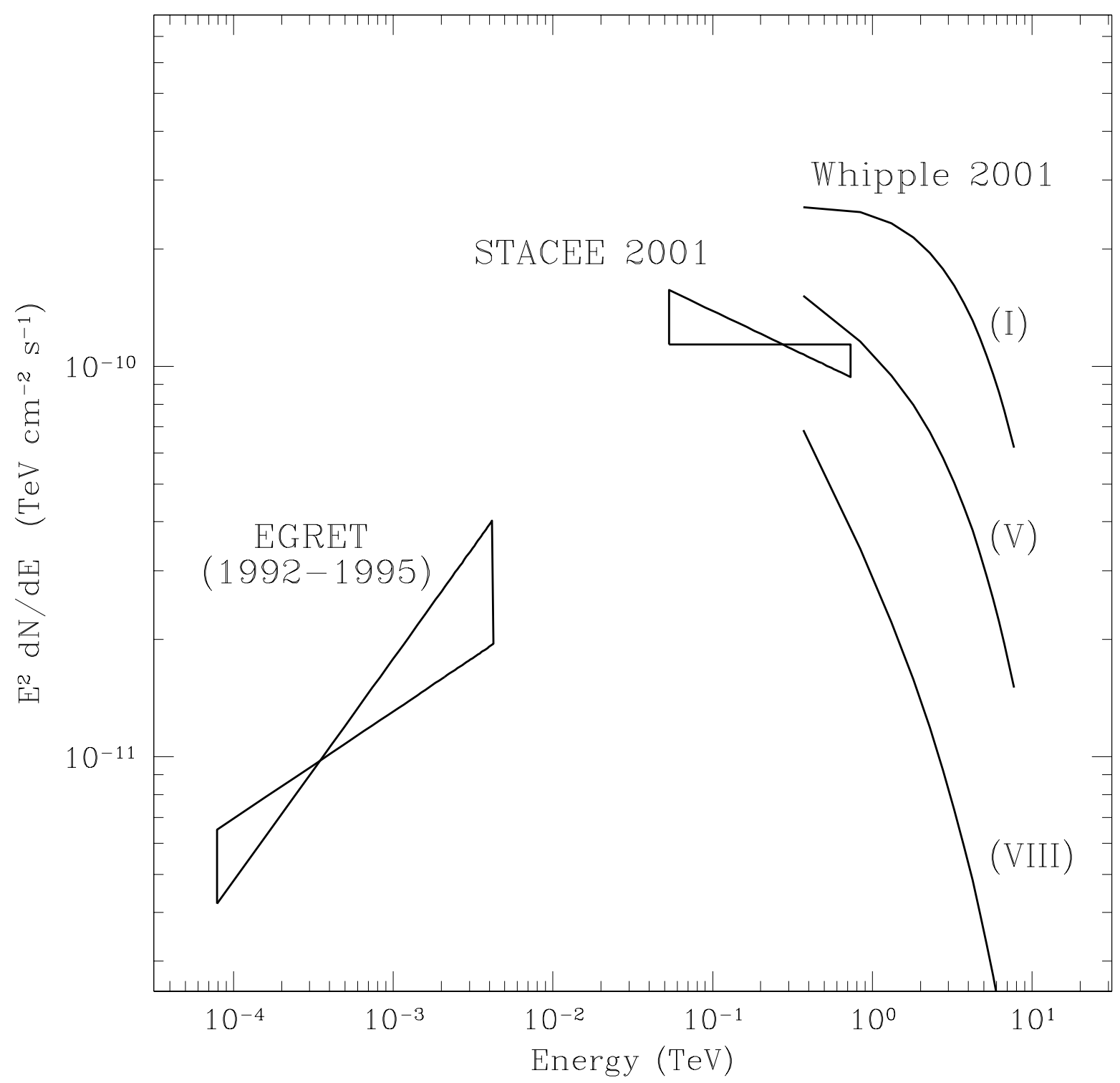

Fig. 2.- High-energy spectrum for Markarian 421. The STACEE butterfly assumes differential spectral indices between $\alpha=2.00$ and 2.20. Note that, though the STACEE sensitivity peaks at $140 \mathrm{GeV}$, the vertex of the butterfly is much higher. STACEE data are averaged over the flaring period, while the Whipple curves represent high (I), medium (V), and low (VIII) flux levels over the course of the flare (see Krennrich et al. 2002, for details). EGRET data are from cycles 1 through 5 , and thus represent an average low state. 


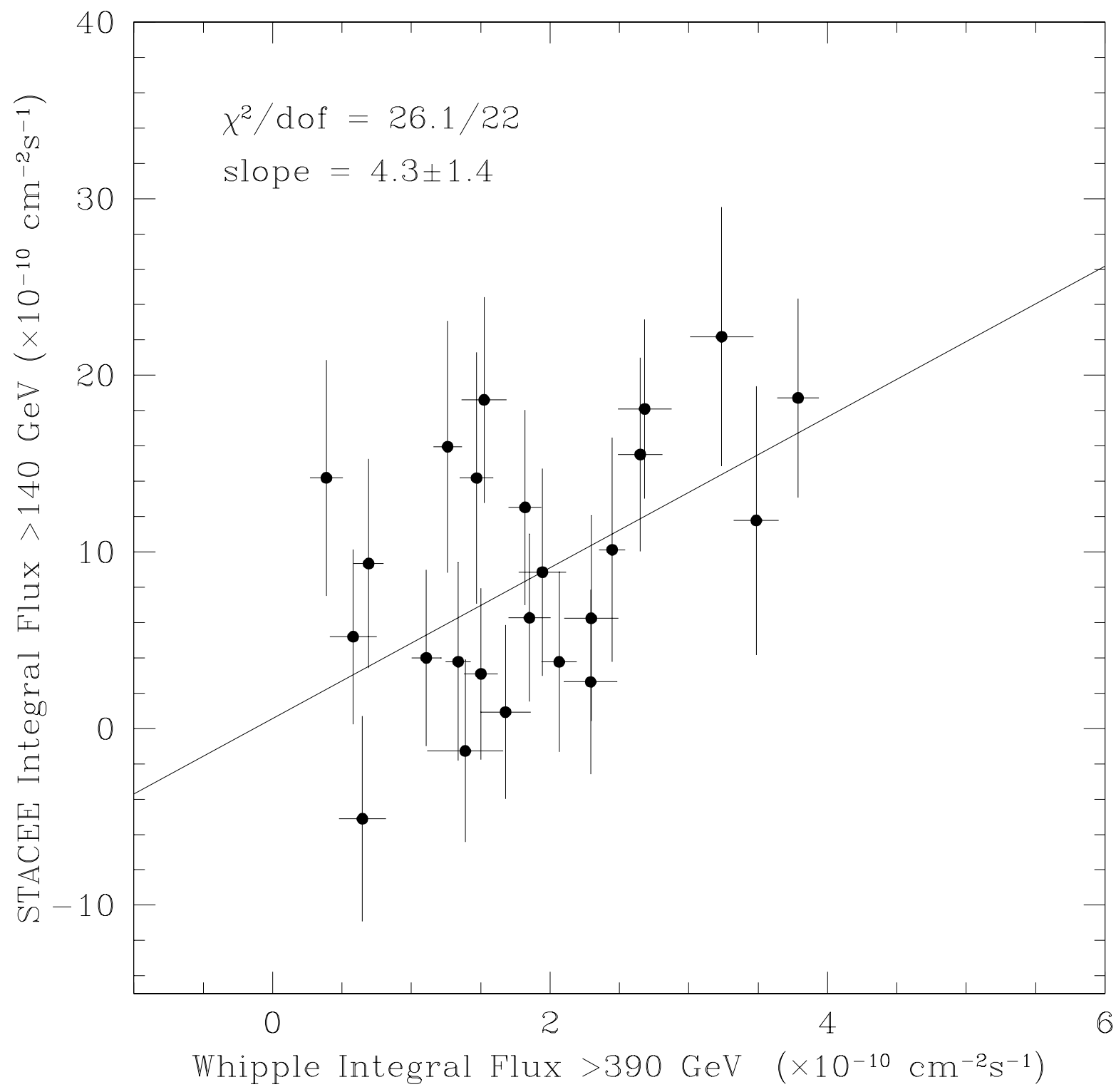

Fig. 3.- Correlations between contemporaneous STACEE and Whipple observations from March to May of 2001. Points represent STACEE and Whipple runs whose time stamps match to within 0.02 days (29 minutes). The $\chi^{2} /$ dof for the fit is $26.1 / 22$. The slope of 4.27 is consistent with a differential spectral index of $\alpha=2.14$, given the STACEE and Whipple thresholds. 

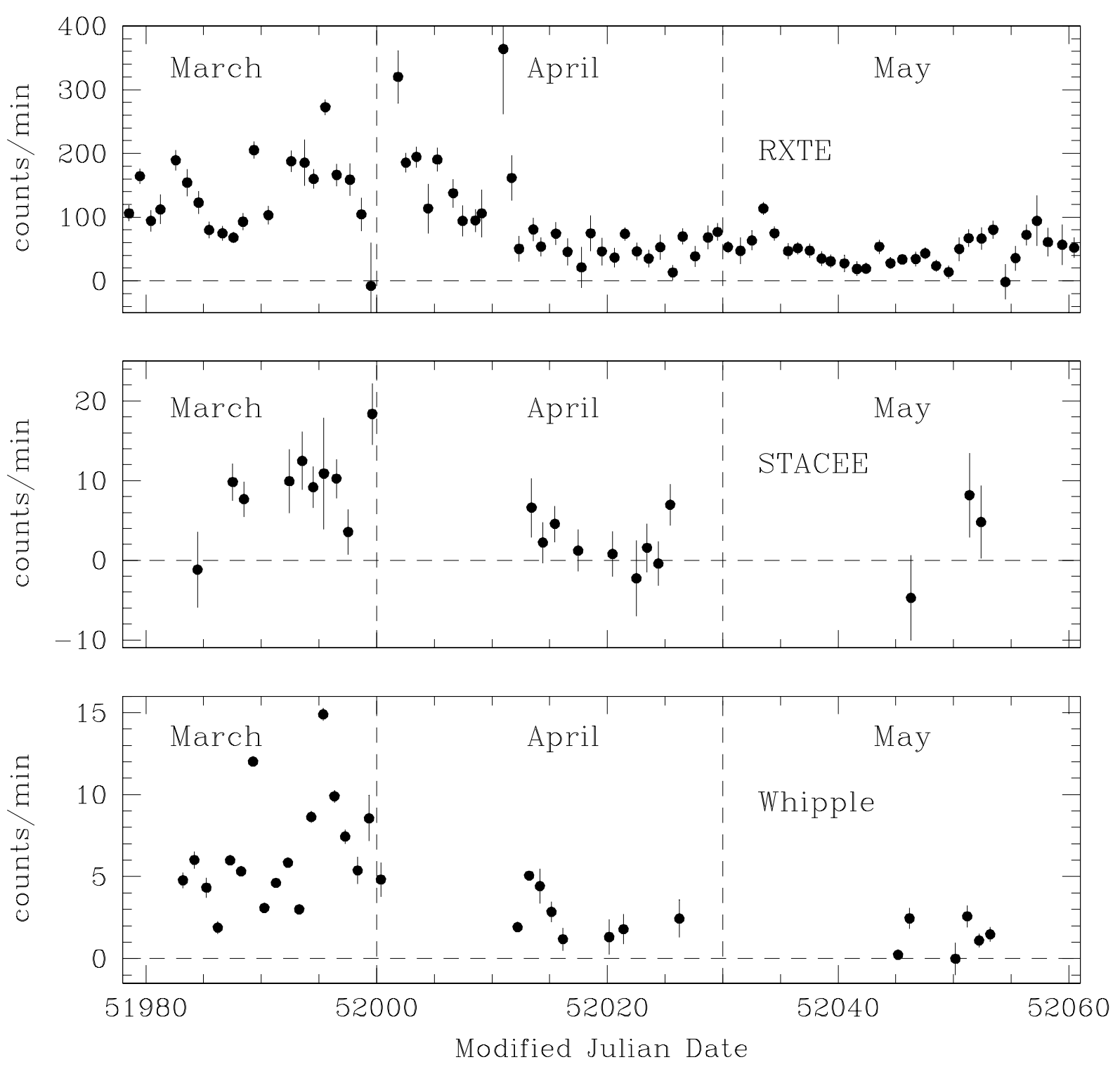

Fig. 4.- Markarian 421 light curve for Spring, 2001. RXTE observations are from the 2-10 $\mathrm{keV}$ band, STACEE data are from the 50-300 GeV band, and Whipple observations span the $0.25-8 \mathrm{TeV}$ band. References cited in the text. 